\title{
La transformación digital en el sector cooperativo agroalimentario español: sítuación y perspectivas
}

\author{
Javier Jorge Vázquez \\ $M^{a}$ Peana Chivite Cebolla \\ Francisco Salinas Ramos
}

RESUMEN: La incorporación de nuevas tecnologías digitales a los procesos de gestión empresarial desempeña hoy en día un factor competitivo determinante en la estrategia de cualquier entidad. Las cooperativas agroalimentarias no pueden quedar al margen de esta oportunidad y, en consecuencia, deben afrontar la transformación digital de sus estructuras no solo para crecer sino también para sobrevivir en un entorno cada vez más cambiante y competitivo. En este trabajo se presenta un estudio exploratorio cuyo objetivo principal se centra en el análisis del uso e implementación de las nuevas tecnologías digitales en las cooperativas agroalimentarias españolas con la finalidad de determinar su grado de transformación digital desde una doble perspectiva: su presencia en internet y los servicios web ofrecidos. Para tal fin, se procede a la revisión de los antecedentes de investigación y posterior estudio centrado en el análisis de un conjunto amplio de parámetros e indicadores sobre el uso de TIC y comercio electrónico que permita determinar la situación del sector cooperativo agroalimentario respecto del conjunto de empresas españolas y realizar un análisis comparativo tanto a nivel territorial como intersectorial. Los resultados obtenidos revelan la existencia de cierto retraso en el grado de transformación digital de las cooperativas agroalimentarias cuya magnitud se encuentra condicionada por el tamaño y el subsector donde desarrollan su actividad.

PALABRAS CLAVE: Agroindustrial, Cooperativas Agrícolas, Adopción de Tecnología, Tecnología y Competitividad, Tecnología de la Información y la Comunicación, Gestión de las TICs, Transformación digital, Empresas de Economía Social, Cooperativas Agroalimentarias.

CLAVES ECONLIT: L660, M150, O330, P130, Q130.

Cómo citar este artículo / How to cite this article: JORGE, J., CHIVITE, Mª.P. \& SALINAS, F. (2019): "La transformación digital en el sector cooperativo agroalimentario español: situación y perspectivas", CIRIEC-España, Revista de Economía Pública, Social y Cooperativa, 95, 39-70. DOI: 10.7203/CIRIEC-E.95.13002.

Correspondencia: Javier Jorge Vázquez, contratado doctor, Universidad Católica de Ávila, e-mail: javier.jorge@ucavila.es; Ma Peana Chivite Cebolla, contratada doctor, Universidad Católica de Ávila, mpeana.chivite@ucavila.es, y Francisco Salinas Ramos, director del Máster Universitario en DAES de la Universidad Católica de Ávila, francisco.salinas@ucavila.es. 


\section{EXPANDED ABSTRACT}

\section{Digital transformation in the Spanish agri-food cooperative sector: situation and prospects}

\section{Context}

Nowadays it is an irrefutable fact that economy digitalisation is changing the traditional methods and balances of the current economic and social organisation model. In this context, the incorporation of new digital technologies in the company plays an undeniable competitive role in its strategic business policy. Agri-food cooperatives cannot ignore this opportunity and must face the digital transformation process not only to survive, but mainly to grow in a particularly changing and competitive market.

\section{Objectives}

The aim of this research is to contribute to the analysis of the digital transformation of the economy, paying special attention to Social Economy and, in particular, to the agri-food cooperative sector in Spain as a strategic sector of the country's productive model. Specifically, our main objective is to present an exploratory study on the use that agri-food cooperatives make of new information and communication technologies in two different aspects: Internet presence and web services offered. In light of the results obtained, an inter-territorial and inter-sectorial comparative study is posed as a complementary objective that allows: i) to characterize the degree of maturity of the digitalisation process of the cooperatives under study; ii) to determine their relative positioning within the Spanish business sector; and iii) to identify any conditioning factors that can exert a greater influence on the degree of digital transformation of agri-food cooperative.

\section{Methodology}

The main method used is the analytical empirical method through frequencies and correlations analysis.

The population considered is constituted by the agri-food cooperatives operating in Spain having more than ten partners / employees, whose data date after the year 2000 according to the Orbis database. Out of 6,431 registered cooperatives, there is a population of 506 entities meeting these criteria.

For the selection of the sample under study, we used stratified random sampling proportionate to the Autonomous Community. The study focuses on cooperative companies with more than 10 partners / employees, the main reasons being summarized in two. On the one hand, since it is based on 


\section{LA TRANSFORMACIÓN DIGITAL EN EL SECTOR COOPERATIVO AGROALIMENTARIO ESPAÑOL: SITUACIÓN Y PERSPECTIVAS}

the hypothesis that agri-food cooperatives present a degree of activity digitalisation below other business legal forms, and therefore focusing on larger ones will help us to position ourselves in the bestcase scenario in order to be able to make a more critical analysis. On the other hand, to be able to compare the study with the one presented by the Spanish Statistics Institute (INE), which makes this same differentiation by size.

For a confidence level of $95 \%$ and an error of $7.9 \%$, a sample of 119 cooperatives is obtained. Thus, as Analucia is the community that has the most cooperatives in this sector, it is also the most represented with 20 cooperatives within the sample.

The search and data collection were carried out during the month of May 2018 through direct analysis of the contents and design of the Web pages corresponding to each of the 119 food cooperatives making up the sample under study. In particular, 11 variables total have been verified.

Three hypotheses will be established to respond to the objectives set:

- Hypothesis $1(\mathrm{H} 1)$ : The degree of digitalisation of agri-food cooperatives in Spain is below that of the business sector as a whole.

- Hypothesis $2(\mathrm{H} 2)$ : The size of the cooperative exerts a significant influence on its degree of digital transformation.

- Hypothesis $3(\mathrm{H} 3)$ : The activity sub-sector determines the level of digitalisation of the agri-food cooperative

\section{Results}

As for the results obtained, the analysis shows that only $55.46 \%$ of the agri-food cooperatives under study have a corporate website. Therese results, when compared with all companies -whose percentage rises to $77.69 \%$-, evidences the existence of some delay in the agri-food cooperative sector in terms of internet presence compared with the average result achieved by the Spanish business sector. Note the existence of a differential greater than 22 percentage points.

The most significant point is to verify that the average in the digitalisation level is 2.3 out of 11 , being this the maximum reachable value. That is, it would not pass on a scale from 0 to 10 . In fact, no cooperative has the highest score possible, being 8 the highest value obtained. We can intuit thanks to the media that there is still much to be done regarding the level of digital transformation.

On another note, the variables that have a higher level of correlation with the variables measuring the level of digital transformation are: operating revenue, partners / number of employees and total assets. Therefore, the three variables established to measure the size of the cooperative influence the level of digital transformation, measured by the 11 items. 
To contrast the third working hypothesis, the sample has been divided into the proposed subsectors following the Spanish Classification of Economic Activities (CNAE-2009). In this case, the outstanding role of the "dairy products" sub-sector, whose cooperatives obtain an average value of 4.67 at the digitalisation level, is verified. On the other hand, $100 \%$ of the cooperatives that operate in this subsector have a website.

Other sub-sectors that do not go unnoticed are "olive oil" and "animal feed products". In both cases, cooperative companies operating in this activity field that have a website represent a significant percentage of $82 \%$ and $71 \%$ respectively. In particular, if we compare these figures with the average of all companies in Spain, we can say that only the "dairy products" and "olive oil" sub-sectors have a percentage of companies with a website that is above the national average.

On the other hand, the activity sub-sectors that reach a higher score in the posed digitalisation index are, in descending order, "dairy products" (4.67 points), "olive oil" (3.25 points), "meat industry" (2.92 points) and "winemaking" (2.89 points). Although all these sub-sectors present a score higher than the average of the agri-food cooperatives analysed, this rating is far from being considered optimal, which indicates the need to promote measures that intensify the process of digital transformation of the agri-food cooperative sector.

\section{Practical conclusions and original value}

Regarding conclusions, after contrasting the formulated hypotheses, it is verified that the digitalisation degree of the agri-food cooperatives is relatively far, in general terms, from the average digitalisation level observed in all Spanish companies, presenting some "delay" in the digital transformation process. Nevertheless, this deficit position does not appear homogeneous in all sub-sectors that make up the agri-food sector. On the contrary, it is verified that both the activity sub-sector and the organisational dimension are determining factors in the digital transformation process of agri-food cooperatives.

Thus, the results obtained reveal that cooperatives with a greater dimension are characterised by presenting a greater digitalisation degree in terms of website development, internet presence, ecommerce and web services offered. Likewise, the dairy, olive oil, wine and meat industries present a global level of digitalisation greater than the rest of the agri-food sub-sectors. Finally, it has not been identified that the territory where the cooperatives carry out their activity exerts any influence on a greater or lesser degree of business digitalisation.

KEYWORDS: Agroindustrial, Agricultural Cooperative, Technology Adoption, Technology and Competitiveness, Information and communication technology, IT Management, Digital Transformation, Social Economy Enterprises, Agri-food Cooperatives. 


\section{LA TRANSFORMACIÓN DIGITAL EN EL SECTOR COOPERATIVO AGROALIMENTARIO ESPAÑOL: SITUACIÓN Y PERSPECTIVAS}

\section{Introducción'}

En la actualidad, la sociedad se encuentra inmersa en un escenario caracterizado por una continua y acelerada transformación impulsada por la concurrencia de varios factores como la globalización, el cambio climático, la innovación tecnológica, la escasez de recursos o los cambios demográficos, tendencias que presionan con vigor hacia una transformación de las formas y equilibrios tradicionales de organización económica y social. En este contexto el proceso de transformación digital se erige como un elemento catalizador y a la vez acelerador de muchos de estos cambios. La irrupción en los últimos años de las nuevas tecnologías digitales, entorno a la cuales pivota este fenómeno, supone hoy en día el pilar fundamental sobre el que se apoyan la mayoría de las innovaciones tecnológicas con especial repercusión en todas las esferas de la sociedad y, por extensión, de la economía.

El acelerado ritmo de desarrollo de las infraestructuras digitales unido a un acceso e interconexión a la red cada vez más rápido y extendido entre los ciudadanos, empresas e instituciones está configurando un ecosistema digital que impulsa numerosos procesos de disrupción y transformación en todos los sectores productivos de la economía, al extremo de vislumbrarse un cambio eminente de los modelos de negocio y del patrón de crecimiento económico en nuestro país.

Desde el punto de vista económico, un estudio recientemente publicado sobre la digitalización de la economía española (Zamora y Arrufi, 2017) revela que en la actualidad la economía digital representa el $22,5 \%$ de la economía munidal, cifra que se preveé crezca al $25 \%$ en el año 2020 . En el caso de España, el procentaje del PIB que puede ligarse actualmente a entornos digitales se aproxima al $20 \%$, lo que equivale a 205 mil millones de euros. Según esta investigación se estima que para 2020 ese porcentaje puede incrementarse hasta el $21,6 \%$, y en el mejor de los casos, hasta el $24 \%$ del PIB siempre que se aplique una combinación eficiente de políticas estratégicas en el ámbito del capital humano, la inversión tecnológica y otros aceleradores digitales.

En este contexto incierto e inestable, las entidades de Economía Social y, en particular, las cooperativas agroalimentarias, deben desarrollar una estrategia integral que les permita transitar de manera ágil y eficiente hacia una economía digital, aprovechando así las ventajas competitivas que surgen en el actual mercado global. Para ello resulta indispensable que las cooperativas adopten un proceso evolutivo de cambio permanente que les permita introducir innovaciones técnicas y organizativas de forma contínua (Marcuello y Sanz, 2008), al tiempo, que propicie ganancias en términos de competitividad y productividad.

1.- El presente trabajo queda enmarcado dentro de un proyecto de investigación de mayor amplitud denominado "Estudio sobre el grado de transformación digital en el sector cooperativo agroalimentario en España: principales retos y oportunidades", financiado por la Asociación de Amigos de la Universidad Católica de Ávila. 
Con la presente investigación se pretende contribuir al análisis de la transformación digital de la economía, prestando especial atención a la Economía Social y, en particular, al sector cooperativo agroalimentario en España, como sector estratégico del modelo productivo del país. En concreto, se plantea como objetivo principal un estudio exploratorio sobre el uso que las cooperativas agroalimentarias realizan de las nuevas tecnologías de la información y la comunicación en dos vertientes diferentes: presencia en internet y servicios web ofrecidos. Para la consecución de este objetivo, se propone el análisis de un conjunto amplio de parámetros ${ }^{2}$ relativos al uso de las TICs y comercio electrónico aplicados a una muestra representativa formada por 119 cooperativas. A la luz de los resultados obtenidos se plantea, como objetivo complementario, un estudio comparativo interterritorial e intersectorial que permita: i) caracterizar el grado de madurez del proceso de digitalización de las cooperativas objeto de estudio; ii) determinar su posicionamiento relativo dentro del sector empresarial español; $y$ iii) identificar aquellos factores condicionantes que pueden ejercer una mayor influencia sobre el grado de transformación digital de las empresas cooperativas agroalimentarias.

El método utilizado es el empírico analítico, a través del análisis de frecuencias y correlaciones. Para la selección de la muestra objeto de estudio se utiliza el muestreo probabilístico aleatorio estratificado proporcionado en función de la comunidad autónoma.

El trabajo que se presenta se articula entorno a la siguiente estructura. En primer lugar se procede a desarrollar una revisión de los antecedentes de la investigación en torno al estado de la cuestión. A la luz de dicha revisión y, tras la determinación de los objetivos del estudio, se formulan tres hipótesis de investigación, a partir de cuya contrastación se procederá al análisis y discusión de los resultados de la investigación y a la extracción de las correspondientes conclusiones del trabajo.

\section{Estado de la cuestión: antecedentes de la investigación e hipótesis de trabajo}

En relación a los antecendetes y al estado actual de los conocimientos, existen diversos autores que han señalado la influencia significativa que ejercen las nuevas tecnologías de la información y la comunicación sobre la mejora de la eficiencia empresarial (Evan y Wurster, 1997; Gosh, 1998; García Canal, 2007; Bernal, et al (2017a, 2018)), el crecimiento económico (Benega, 2003; Mas y Quesada, 2005; Jorgenson, et al., 2007; Zamora, 2016; Zamora y Arrufi, 2017), y las ganancias en la productividad de los factores productivos en las empresas (Nordhaus, 2002; Bresnahan et al., 2003; Hernando

2.- En concreto, se tomarán ítems similares a los empleados por el Instituo Nacional de Estadística en su "Encuesta de uso de TIC y Comercio Electrónico (CE) en las empresas 2016-2017" con la finalidad de desarrolalr un análisis comparativo y determinar el posicionamiento relativo de las cooperativas agroalimentarias respecto al conjunto de empresas españolas. 


\section{LA TRANSFORMACIÓN DIGITAL EN EL SECTOR COOPERATIVO AGROALIMENTARIO ESPAÑOL: SITUACIÓN Y PERSPECTIVAS}

y Nuñez, 2004; Atrostic y Nguyen, 2005; Van Reenen et al., 2007; Torrent, et al., 2008; Ontiveros y Vizcaíno, 2017).

Si bien en los últimos años han proliferado estudios e informes relativos al uso de las nuevas tecnologías de la información y la comunicación (TIC) en el tejido empresarial español (ONTSI y FUNDETEC, 2014; CES, 2017; Zamora y Arrufí, 2017; entre otros) no son muchos los trabajos específicos que se han encontrado dedicados al estudio de la transformación digital de las entidades de Economía Social. Los escasos trabajos desarrollados en dicha área se han centrado principalmente en el sector no lucrativo, mientras que el resto giran en torno a estudios descriptivos y exploratorios sobre las oportunidades que brindan las nuevas tecnologías en áreas específicas de la organización de empresa como la gestión comercial, la comunicación de redes, la predisposición a la innovación y los procesos de internacionalización. A continuación se recogen la finalidad y principales resultados obtenidos de dichos trabajos aplicados al sector cooperativo agrario-agroalimentario:

Uno de los trabajos pioneros en analizar las oportunidades que el uso de internet brindaba al cooperativismo agrario español fue el desarrollado por Mozas, et al. (2001). En dicha investigación se analizaban las distintas posibilidades de aplicación de internet en la gestión empresarial y, en particular, en la gestión comercial, especialmente en el sector cooperativo oleícola. Además se ofrecía un estudio descriptivio sobre el grado de implantación las nuevas tecnologías en el sector que confirmaba su escasa aplicación e identificaba las principales barreras para su adopción.

Mozas et al. (2004) emprende un estudio sobre el sector cooperativo español de segundo grado cuya finalidad es analizar la situación y perspectivas de futuro en cuanto a la utilización de internet y, en particular, del comercio electrónico. Los resultados obtenidos de la investigación revelan que, si bien el grado de conexión a internet se ecuentra por encima de la media del resto de empresas españolas, el desarrollo e implantación del comercio electrónico presenta unos resultados que animan a incrementar significativamente los esfuerzos de todo el sector (tan solo un 7\% de las cooperativas analizadas disponen de un canal de venta online y únicamente el $9 \%$ compran a sus proveedores a través de internet).

En la investigación desarrollada por Espasandín, et al. (2004) sobre grupos estratégicos se analiza el impacto que la incorporación de nuevas tecnologías de la información y la comunicación genera en los procesos de innovación e internacionalización de las entidades de la Economía Social en la Comunidad Autónoma de Andalucía. Las conclusiones a las que llegan en su estudio señalan que el posicionamiento de las empresas respecto a estas tenologías condicionan su predisposición a la innovación presente y futura y a sus expectativas sobre los procesos de internacionalización.

Por su parte, Juliá, et al. (2004) presentan un estudio descriptivo sobre el uso de páginas web por parte de empresas cooperativas pertenecientes a distintos sectores productivos, centrando la atención en el tipo de información divulgada y en los servicios y contenidos recogidos. Los resultados obtenidos apuntan la necesidad de incidir en el desarrollo tanto de los contenidos generales, como de los contenidos específicos relacionados con su realidad y dimensión social. 
El binomio nuevas tecnologías-ventaja competitiva ha sido objeto de especial atención en la literatura económica de los últimos años. En este contexto, el trabajo de Vargas (2004) examina dicha relación centrando la atención en las empresas cooperativas, analizando la vinculación existente entre la obtención de ventajas competitivas y la implementación de las nuevas tecnologías de la información desde la perspectiva de la Teoría de los Costes de Transacción.

Meroño et al. (2006) llevan a cabo un trabajo que tiene por finalidad conocer tanto el grado de equipamiento tecnológico como el patrón de utilización de las tecnologías en la gestión de la información en el sector cooperativo hortofrutícola de la Región de Murcia. Los datos recopilados demuestran que las cooperativas agrarias, lejos de la imagen tradicional de "retraso tecnológico", presentan un buen posicionamiento en términos de equipamiento y uso de aplicaciones informáticas de gestión, si bien en términos de aprovechamiento y presencia en internet aún muestran ciertas carencias respecto del resto de empresas competidoras.

Bernal et al. (2008) desarrollan un estudio longitudinal cuya finalidad es diagnósticar el uso comercial que las cooperativas de segundo grado españolas realizan de sus sitios webs. A partir de una amplia muestra y a través de la observación directa de un conjunto de parámetros y dimensiones concluyen que existe una deficitaria calidad de la información suministrada y del sistema, si bien se observa una evolución positiva durante los últimos años.

Mozas et al. (2008) presentan un trabajo de campo cuyo objetivo es determinar el nivel de utilización de internet en la gestión comercial de las cooperativas agrarias que operan en el sector oleícola jienense. De los resultados obtenidos se desprende que, si bien el grado de conectividad a la red se encuentra en consonancia con el resto de empresas españolas, la calidad de dicha conexión y la intensidad en su uso para fines comerciales presentan un cierto retraso con respecto a otros sectores empresariales.

Por otro lado, López et al. (2012) realizan un estudio exploratorio sobre el grado de utilización de los sitios web en el sector cooperativo agroalimentario, analizando los factores que condicionan su adopción y sus consecuencias. Los resultados de este trabajo vinculan la utilización de sitios Web con el grado de complejidad percibida en su uso, el nivel de formación del equipo directivo o con la antigüedad de la empresa.

Montegut et al. (2013) desarrolla un análisis sobre el uso de internet y el comercio electrónico en el sector cooperativo oleícola y frutícola de la provincia de Lleida. Los hallazgos alcanzados revelan que, si bien existe un elevado nivel de equipamiento informático y conectividad de las entidades analizadas, presentan carencias reseñables en términos de comercio electrónico, presencia en internet y uso de la red como medio para el establecimiento de relación con clientes y proveedores.

Por su parte, Fernández et al. (2016) llevan a cabo un estudio empírico sobre el grado de utilización del comercio electrónico y de las redes sociales en el sector oleícola andaluz, aplicando para ello 


\section{LA TRANSFORMACIÓN DIGITAL EN EL SECTOR COOPERATIVO AGROALIMENTARIO ESPAÑOL: SITUACIÓN Y PERSPECTIVAS}

un enfoque comparativo entre las cooperativas y otras formas jurídicas que operan en dicho sector. Los resultados señalan el uso limitado de los sistemas electrónicos y telemáticos en la gestión comercial, y el reducido número de empresas que utilizan las redes sociales. Además se constata una posición peor de las cooperativas, en comparación con otras formas jurídicas empresariales, tanto en el uso como en la implementación de las TIC en la gestión empresarial.

Bernal et al. (2017a) desarrollan un análisis sobre la eficiencia económica del sector productivo de aceite de oliva ecológico en España con la finalidad de identificar los principales factores explicativos de las mejores prácticas organizativas. Los resultados evidencian la existencia de bajos niveles de eficiencia económica en este sector y señalan al compromiso por las ventas online, las heramientas web, la formación, las exportaciones y el tamaño de las organizaciones, como variables que combinadas impulsan la eficiencia económica de estas organizaciones.

Bernal et al. (2018) llevan a cabo un estudio cuyo objetivo es examinar la relación existente entre la eficiencia económica de las compañías de aceite de oliva orgánico en España y la calidad de sus sitios web corporativos, así como identificar las principales variables que pueden mejorar dicha relación. Si bien los resultados obtenidos en este estudio no identificaron la existencia de una relación directa entre estas variables, sí que revelaron que la combinación de determinados parámetros relacionados con la calidad del portal web y otras variables estructurales y organizativas pueden tener un impacto directo sobre el desarrollo de la organización en términos de eficiencia, concluyendo que las TIC son un factor significativo para mejorar la competitividad y desarrollo del sector olivarero español.

Si atendemos a las fuentes estadísticas sobre el grado de transformación digital en el sector empresarial español cabe destacar la "Encuesta de uso de TIC y Comercio Electrónico" que desde el año 2001 viene desarrollando con una periodicidad anual el Instituto Nacional de Estadística (INE). En dicha encuesta se emplean un conjunto amplio de variables sobre uso de TIC, comercio electrónico y formación en TIC. Los últimos datos publicados correspondientes al periodo 2016-17 (INE, 2017) señalan que:

- El $73,1 \%$ de las empresas con menos de 10 empleados poseen equipos informáticos y el $70,2 \%$ tienen conexión a internet, de las cuáles el 29,8\% dispone de página web.

- Las empresas con 10 o más empleados que dispone de conexión a internet representan el $98,7 \%$, magnitud que se reduce al $82,4 \%$ si se considera la conexión via banda ancha. Aproximadamente el $80 \%$ además tienen página web. El 31,4\% realizan compras por comercio electrónico y una de cada cinco realizaron ventas a través de comercio electróncio. Por sectores, los resultados relativos a los servicios web aparecen recogidos en la tabla 1: 


\section{Tabla 1. Encuesta de uso de TIC y Comercio Electrónico en las empresas 2016-2017 (empresas con más de 10 empleados): Servicios / Página Web}

\begin{tabular}{|l|c|c|c|c|}
\hline TOTAL NACIONAL: Servicios Web & Total Empresas & Industria & Construcción & Servicios \\
\hline $\begin{array}{l}\text { D.7.1.J: Posibilidad de envío electrónico de hojas } \\
\text { de reclamaciones (2) }\end{array}$ & 27,78 & 25,59 & 20,95 & 30,12 \\
\hline $\begin{array}{l}\text { D.7.1.I: Anuncios de ofertas de trabajo o recepción } \\
\text { de solicitudes de trabajo online (2) }\end{array}$ & 21,01 & 13,67 & 14,70 & 25,60 \\
\hline $\begin{array}{l}\text { D.7.1.H: Declaración de política de intimidad o } \\
\text { certificación relacionada con la seguridad del sitio } \\
\text { Web (2) }\end{array}$ & 71,08 & 66,37 & 63,93 & 74,63 \\
\hline $\begin{array}{l}\text { D.7.1.G: Vínculos o referencias a los perfiles de } \\
\text { la empresa en medios sociales (2) }\end{array}$ & 46,72 & 37,47 & 33,29 & 53,58 \\
\hline $\begin{array}{l}\text { D.7.1.F: Personalización de la página Web } \\
\text { para usuarios habituales (2) }\end{array}$ & 7,83 & 5,53 & 6,73 & 9,10 \\
\hline D.7.1.E Seguimiento online de pedidos (2) & 10,90 & 6,18 & 1,66 & 14,86 \\
\hline $\begin{array}{l}\text { D.7.1.D: Posibilidad de personalizar o diseñar los } \\
\text { productos por parte de los clientes (2) }\end{array}$ & 8,15 & 6,51 & 1,57 & 10,18 \\
\hline $\begin{array}{l}\text { D.7.1.C: Acceso a catálogos de productos o a } \\
\text { listas de precios (2) }\end{array}$ & 49,42 & 55,07 & 24,04 & 51,77 \\
\hline D.7.1.B: Recepción de pedidos o reservas online(2) & 18,00 & 11,78 & 2,83 & 23,80 \\
\hline $\begin{array}{l}\text { D.7.1.A: Presentación de la empresa (2) } \\
\text { D.7 \% de empresas con conexión a Internet y } \\
\text { sitio/página Web (1) }\end{array}$ & 89,57 & 89,96 & 90,80 & 89,16 \\
\hline
\end{tabular}

(1) Porcentaje sobre el total de empresas con conexión a Internet.

(2) Porcentaje sobre el total de empresas con conexión a Internet y página Web.

FUENTE: INE (2017).

Tal y como se señaló, si bien es cierto que existen trabajos sobre la transformación digital a nivel nacional para empresas mercantiles, se han encontrado menos referencias en el ámbito de las empresas cooperativas, siendo el sector oleícola y frutícola, o las cooperativas de segundo grado, el que presenta el mayor número. Por tanto, con este trabajo se quiere contribuir a ampliar este tipo de estudios. Se utilizará como fuente estadística de referencia la "Encuesta de uso de TIC y Comercio Electrónico" del INE (2017) para poder contrastar los datos recopilados en nuestro estudio y desarrollar un análisis comparativo, que permita determinar el grado de transformación digital de las cooperativas agroalimentarias y su posicionamiento relativo respecto del conjunto de empresas en España. Se incluirá también un análisis complementario por subsectores. 


\section{LA TRANSFORMACIÓN DIGITAL EN EL SECTOR COOPERATIVO AGROALIMENTARIO ESPAÑOL: SITUACIÓN Y PERSPECTIVAS}

Se parte de la hipótesis de que las cooperativas agroalimentarias presentan un grado de digitalización de su actividad menor que otras formas jurídicas empresariales. Es por ello que el estudio se centra en las empresas cooperativas con más de 10 socios / empleados, puesto que serán las que presentan un mayor nivel de digitalización. Y en esta línea se planteará la primera hipótesis.

Por otro lado, se quiere contrastar cuales son los factores condicionantes en el proceso de digitalización de las cooperativas agroalimentarias. Al igual que otros trabajos coinciden en señalar que el tamaño es una variable determinante a la hora de implantar ciertas políticas (Chivite et al, 2014; Bernal et al., 2017b; Bernal et al., 2018) parece apropiado tomar también este tipo de variables de forma complementaria para enriquecer la aportación. Trabajos previos como el de López et al. (2012), como se ha señalado, vinculan la utilización de sitios Web con el grado de complejidad percibida en su uso, el nivel de formación del equipo directivo o con la antigüedad de la empresa, pero no con el tamaño ni el subsector de actividad. En esta línea se plantea la segunda y tercera hipótesis.

Una vez planteado el estado de la cuestión, se formulan las siguientes hipótesis de investigación:

- Hipótesis 1 (H1): El grado de digitalización de las cooperativas agroalimentarias en España es menor que en el sector empresarial en su conjunto.

- Hipótesis 2 (H2): El tamaño de la cooperativa ejerce una influencia significativa sobre su grado de transformación digital.

- Hipótesis 3 (H3): El subsector de actividad condiciona el nivel de digitalización de la cooperativa agroalimentaria.

\section{Metodología}

Tras la revisión de los antecedentes de investigación, para el estudio del caso que nos ocupa, se va a emplear principalmente el método empírico analítico, a través del análisis de frecuencias y correlaciones. 
La población que ha sido considerada está constituida por las cooperativas agroalimentarias activas en España, con más de diez socios / empleados ${ }^{3}$, cuyos datos fueran posteriores al año $2000^{4}$ según la base de datos $\mathrm{Orbis}^{5}$. De un total de 6.431 cooperativas registradas, queda una población conforme a estos criterios de 506 entidades.

Para la selección de la muestra objeto de estudio se utiliza el muestreo probabilístico aleatorio estratificado proporcionado en función de la comunidad autónoma ${ }^{6}$, según se puede observar en la tabla 2, y cuyo procedimiento se explica a continuación.

Aplicando el muestreo probabilístico ${ }^{7}$ aleatorio estratificado proporcionado (Hair, 2010) en función de la comunidad autónoma, para un error de muestreo del 7,9\%

$$
n=\frac{N \times Z_{a}^{2} \times p \times q}{d^{2} \times(N-1)+Z_{a}^{2} \times p \times q}
$$

$\mathrm{N}$ = tamaño de la población, que será de 506

$Z=$ nivel de confianza, para el $95 \%$ será 1.96

$\mathrm{p}=$ probabilidad de éxito, o proporción esperada, se le da un 0.5 , dato para la mayor dispersión

$q=$ probabilidad de fracaso $(1-p)$

$\mathrm{d}=$ precisión (Error máximo admisible en términos de proporción), del 7,9\%8

$$
\begin{gathered}
\frac{506 * 1,96^{2} * 0.5 * 0.5}{0,079^{2} *(506-1)+1,96^{2} * 0.5 * 0.5}=118,17 \\
\frac{N i * n}{N}
\end{gathered}
$$

3.- La razón por la cual el estudio se centra en las empresas cooperativas con más de 10 socios / empleados, radica, por un lado, en que se parte de la hipótesis de que las cooperativas agroalimentarias presentan un grado de digitalización de su actividad menor que otras formas jurídicas empresariales y, por tanto, centrarnos en las de mayor tamaño, nos ayudará a posicionarnos en el mejor de los casos, para poder hacer un análisis más crítico. Por otro lado, para poder comparar el estudio con el desarrollado por el INE, que hace esta misma diferenciación por tamaño.

4.- El hecho de que los datos seleccionados fueran posteriores al año 2000 responde al objetivo de reducir la posibilidad de desviación entre la realidad y la información facilitada.

5.- La base de Datos "Orbis" tiene información empresarial a nivel mundial, y pertenece al grupo BvD (Bureau van Dijk) Para la selección de la actividad "Agroalimentaria", la búsqueda ha seguido el siguiente criterio: 01 - Agricultura, ganadería, caza y servicios relacionados con las mismas, 03 - Pesca y acuicultura, 10 - Industria de la alimentación, 11 Fabricación de bebidas. La fecha de consulta ha sido 11/04/2018.

6.- La aplicación del muestreo probabilístico aleatorio estratificado proporcionado en función de la comunidad autónoma permite que todas las regiones estén representadas, pero aquellas con mayor número de cooperativas en mayor medida, lo que permitirá realizar un análisis interterritorial adicional.

7.- Referencias de consulta para decidir establecer este muestreo son por ejemplo Hair (2010).

8.- El nivel de error utilizado, sin llegar al 10\%, permite revisar el 23,5\% de las cooperativas, lo que consideramos es representativo para este estudio, puesto que en la mayoría de las variables la probabilidad $p$, no será del 0,5 . 
Para calcular el tamaño de la muestra para cada comunidad se emplea la siguiente expresión:

Siendo $\mathrm{Ni}=$ tamaño de la población en la comunidad autónoma i

$\mathrm{n}=$ tamaño de la muestra 9

$\mathrm{N}=$ Población total, los 506

\section{Tabla 2. Muestra de cooperativas por comunidad}

\begin{tabular}{|c|c|c|}
\hline Comunidad autónoma & $\mathrm{N}^{\circ}$ cooperativas & Tamaño de la muestra \\
\hline 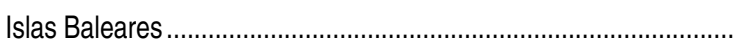 & 1 & 1 \\
\hline Cantabria & 2 & 2 \\
\hline 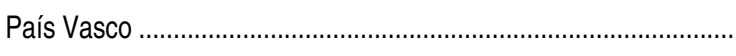 & 7 & 2 \\
\hline Madrid & 7 & 2 \\
\hline Asturias & 10 & 2 \\
\hline Navarra & 11 & 3 \\
\hline 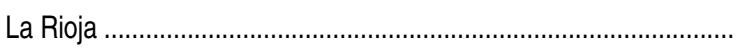 & 13 & 3 \\
\hline 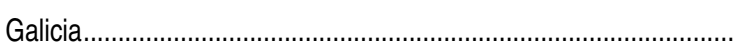 & 15 & 3 \\
\hline Islas Canarias & 20 & 5 \\
\hline 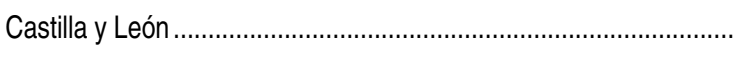 & 24 & 6 \\
\hline Murcia & 30 & 7 \\
\hline Cataluña & 48 & 11 \\
\hline Aragón & 49 & 11 \\
\hline 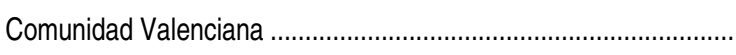 & 51 & 12 \\
\hline Extremadura & 62 & 14 \\
\hline Castilla-La Mancha & 67 & 15 \\
\hline (1) & 89 & 20 \\
\hline TOTAL.. & 506 & 119 \\
\hline
\end{tabular}

FUENTE: Elaboración propia.

9.- Frente a las 119, $n$ se sustituye por 116. Así, para las Islas Baleares se ha seleccionado por defecto la cooperativa que aparece 1, lo mismo para Cantabria, las 2 que aparecen. Por tanto, el resto de comunidades autónomas ponderan sobre 119-3=116. Esto para asegurar la representatividad de todas las comunidades autónomas. 


\section{Tabla 3. Servicios ofertados en internet y comercio electrónico: variables seleccionadas}

\begin{tabular}{|c|c|c|}
\hline & Variable & Definición \\
\hline V.0 & $\begin{array}{l}\text { Disponibilidad de sitio/página web de la } \\
\text { cooperativa. }\end{array}$ & $\begin{array}{l}\text { Comprende la existencia de un portal web específico de la empresa cooperativa así } \\
\text { como su posicionamiento en el motor de búsqueda de Google }{ }^{10} \text {. }\end{array}$ \\
\hline V.1 & $\begin{array}{l}\text { Presentación corporativa de la entidad } \\
\text { cooperativa. }\end{array}$ & $\begin{array}{l}\text { Relacionada con la publicación de información suficiente y adecuada sobre la enti- } \\
\text { dad cooperativa y su actividad. }\end{array}$ \\
\hline V.2 & Recepción de pedidos o reservas online. & $\begin{array}{l}\text { Relativa a la existencia de un canal de ventas a través de comercio electrónico. } \\
\text { Se evalúa la existencia de soportes o plataformas de comercio electrónico que per- } \\
\text { mitan la recepción de pedidos, las reservas de bienes o servicios a través de inter- } \\
\text { net u otras redes telemáticas. }\end{array}$ \\
\hline V.3 & $\begin{array}{l}\text { Acceso a catálogos de productos o lis- } \\
\text { tas de precios. }\end{array}$ & $\begin{array}{l}\text { Se refiere a la posibilidad y facilidad de acceso, a través del sitio web, de catálo- } \\
\text { gos de bienes y/o servicios ofertados por la cooperativa o publicación de tarifas de } \\
\text { precios de sus productos. }\end{array}$ \\
\hline V.4 & $\begin{array}{l}\text { Posibilidad de personalización y diseño } \\
\text { de productos por parte de los clientes. }\end{array}$ & $\begin{array}{l}\text { Está relacionado con la inclusión en el proceso de compra de herramientas que per- } \\
\text { mitan al usuario la personalización y/o coparticipación en el diseño de los bienes y } \\
\text { servicios ofrecidos por la cooperativa. }\end{array}$ \\
\hline V.5 & Seguimiento online de pedidos. & $\begin{array}{l}\text { Dotación en la página web de plataformas u otro medio telemático que permita el } \\
\text { seguimiento en tiempo real del estado de tramitación del pedido, desde la finaliza- } \\
\text { ción del proceso de compra online hasta la entrega efectiva del producto al cliente. }\end{array}$ \\
\hline V.6 & $\begin{array}{l}\text { Personalización de la página Web para } \\
\text { usuarios habituales. }\end{array}$ & $\begin{array}{l}\text { Relacionada con la adaptación de los contenidos y estructura de la web en función } \\
\text { del comportamiento observado del usuario, así como de sus atributos específicos } \\
\text { (perfil, localización, etc.) con la finalidad de ofrecer una mejora en la experiencia de } \\
\text { navegación. }\end{array}$ \\
\hline V.7 & $\begin{array}{l}\text { Vínculos o referencias a los perfiles de } \\
\text { las cooperativas en medios sociales. }\end{array}$ & $\begin{array}{l}\text { Presencia en el portal web corporativo de referencias explícitas y links a las princi- } \\
\text { pales plataformas de comunicación que permitan la interacción y el intercambio de } \\
\text { contenido e información con proveedores, clientes y resto de agentes que se rela- } \\
\text { cionan con la actividad de la cooperativa. }\end{array}$ \\
\hline V.8 & $\begin{array}{l}\text { Declaración de política de intimidad o } \\
\text { certificación relacionada con la seguri- } \\
\text { dad del sitio Web. }\end{array}$ & $\begin{array}{l}\text { Inclusión en el sitio web de un apartado específico reservado a la descripción de } \\
\text { la política de privacidad y protección de datos, utilización de la página y limitaciones } \\
\text { de uso, utilización de cookies, seguridad, etc. }\end{array}$ \\
\hline V.9 & $\begin{array}{l}\text { Anuncios de ofertas de trabajo o recep- } \\
\text { ción de solicitudes de trabajo online. }\end{array}$ & $\begin{array}{l}\text { Hace referencia a la utilización de la página web como medio electrónico al servicio } \\
\text { de los procesos de reclutamiento de personal. Se incluyen elementos tales como la } \\
\text { existencia de un tablón de ofertas de empleo, la disponibilidad de un canal habili- } \\
\text { tado para el envío de C.V., etc. }\end{array}$ \\
\hline V.10 & $\begin{array}{l}\text { Posibilidad de envío electrónico de hojas } \\
\text { de reclamaciones. }\end{array}$ & $\begin{array}{l}\text { Existencia en la página web de un canal específico para el envío de reclamacio- } \\
\text { nes o, en su defecto, la publicación en la website de instrucciones específicas para } \\
\text { cursar reclamaciones a través de otros medios telemáticos (por ejemplo: vía email). }\end{array}$ \\
\hline
\end{tabular}

FUENTE: Elaboración propia.

10.- La elección del buscador de Google responde a su posición de dominio en el mercado mundial al representar una cuota de mercado estimada próxima al $60 \%$ según los datos publicados por la consultora Stat Counter (mayo 2018). 


\section{LA TRANSFORMACIÓN DIGITAL EN EL SECTOR COOPERATIVO AGROALIMENTARIO ESPAÑOL: SITUACIÓN Y PERSPECTIVAS}

La búsqueda y recopilación de datos se ha llevado a cabo durante el mes de mayo de 2018 a través del análisis directo del contenido y diseño de las páginas Web ${ }^{11}$ correspondientes a cada una de las 119 cooperativas agroalimentarias que forman parte de la muestra objeto de estudio. En particular, se han verificado un total de 10 variables que se describen a continuación en la tabla 3.

Cada una de las variables (V) podría tomar el valor 1, si posee la característica, 0 de 0 en caso contrario.

Con la finalidad de dar cumplimiento al objetivo de establecer un análisis comparativo del grado de transformación digital entre las cooperativas agroalimentarias y el resto de formas jurídicas empresariales, se ha optado por seleccionar un conjunto de variables que fueran homologables a los ítems recogidos, bajo la categoría de "servicios web", en la "Encuesta de uso de TIC y Comercio Electrónico (CE)"12 elaborada por el Instituto Nacional de Estadística ${ }^{13}$ y que se muestran en la tabla 1.

Para contrastar el conjunto de hipótesis formuladas en la presente investigación se ha optado por la aplicación de los siguientes métodos. En el contraste de la primera hipótesis se utilizará principalmente el análisis de frecuencias de dichas variables. Para contrastar la segunda hipótesis se incorporan al análisis un conjunto de variables adicionales condicionantes del tamaño de la cooperativa (ver tabla 4). En esta etapa del estudio se llevará a cabo un análisis de frecuencias y de correlación entre las variables propuestas.

\section{Tabla 4. Variables adicionales seleccionadas relativas al tamaño de la cooperativa agroalimentaria}

\begin{tabular}{|l|l|}
\hline \multicolumn{2}{|c|}{ Variable } \\
\hline A.0 & Ingresos de explotación (1) \\
A.1 & Número de socios/empleados (1) \\
A.2 & Total de activos (1) \\
\hline
\end{tabular}

Se obtendrá los datos calculados para el último ejercicio disponible ${ }^{14}$ en la base de datos "Orbis". FUENTE: Elaboración propia.

11.- Datos a disposición.

12.- Nótese la existencia de un posible sesgo como consecuencia del desfase temporal existente al contrastarse los últimos datos recopilados por el INE en la mencionada encuesta correspondientes al periodo 2016-17 con los obtenidos en el presente estudio de 2018, siendo que la diferencia pudiera ser algo más apuntada que la que se presenta.

13.- Véase INE (2017): Encuesta de uso de TIC y Comercio Electrónico años 2016-2017, Madrid: Instituto Nacional de Estadística.

14. - El último ejercicio disponible difiere para cada cooperativa, en concreto: Para el año 2016, se disponen de datos de 42 cooperativas, para el año 2015, 22, para 2014, 10, para 2013, 5, para 2012, 6, para 2011, 2007 y 2003 son 4 por año, para 2010, 2009, 2006, 2001 y 2000 son 3, 2017, 2005, 2002 son 2, y 2008 una. 
Para contrastar la tercera hipótesis se establece una clasificación por subsectores y se desarrolla un análisis comparativo intersectorial con la finalidad de determinar si la pertenencia a un subsector determinado de actividad influye sobre el grado de transformación digital de la cooperativa agroalimentaria.

Dado que el sector agroalimentario se puede dividir en dos grandes bloques de actividad: la industria de la alimentación y la fabricación de bebidas, se ha optado por construir, dentro de cada uno de estos dos ámbitos, una clasificación por subsectores tomando como referencia la Clasificación Nacional de Actividades Económicas (CNAE-2009), tal y como puede observarse en la tabla 5.

\section{Tabla 5. Clasificación del sector agroalimentario}

\begin{tabular}{|c|c|c|c|}
\hline $\mathrm{N}^{\circ}$ & Subsectores & $\mathrm{N}^{\circ}$ & Subsectores \\
\hline 1 & Industria cárnica & 8 & Azúcar, chocolate y confitería \\
\hline 2 & Industria del pescado & 9 & Otros productos alimenticios, incluye café e infusiones \\
\hline 3 & Preparación y conservación de frutas y hortalizas & 10 & Productos alimentación animal \\
\hline 4 & Aceites y grasas & 11 & Vinos \\
\hline 5 & Productos lácteos & 12 & Bebidas espirituosas + Otras bebidas alcohólicas \\
\hline 6 & Molinería y almidones & 13 & Agua embotellada y bebidas aromatizadas \\
\hline 7 & Panadería y pastas alimenticias & & \\
\hline
\end{tabular}

FUENTE: Elaboración propia a partir de la Clasificación Nacional de Actividades Económicas CNAE-2009 (INE).

De acuerdo con la clasificación propuesta, en la tabla 6 se presenta la distribución de las cooperativas agroalimentarias que conforman la muestra objeto de estudio. 


\section{Tabla 6. Distribución de la muestra de cooperativas agroalimentarias por subsectores}

\begin{tabular}{|c|c|c|c|c|c|c|}
\hline & & & Frecuencia & Porcentaje & $\begin{array}{l}\text { Porcentaje } \\
\text { válido }\end{array}$ & $\begin{array}{l}\text { Porcentaje } \\
\text { acumulado }\end{array}$ \\
\hline \multirow[t]{10}{*}{ Válidos } & 1 & Industria cárnica & 12 & 10,1 & 10,5 & 10,5 \\
\hline & 3 & $\begin{array}{l}\text { Preparación y conservación de frutas y hortalizas. } \\
\text { (hortofrutícola) }\end{array}$ & 33 & 27,7 & 28,9 & 39,5 \\
\hline & 4 & Aceites y grasas (oleícola) & 12 & 10,1 & 10,5 & 50,0 \\
\hline & 5 & Productos lácteos & 6 & 5,0 & 5,3 & 55,3 \\
\hline & 7 & Panadería y pastas alimenticias. & 2 & 1,7 & 1,8 & 57,0 \\
\hline & 9 & Otros productos alimenticios, incluye café e infusiones. & 16 & 13,4 & 14,0 & 71,1 \\
\hline & 10 & Productos alimentación animal & 14 & 11,8 & 12,3 & 83,3 \\
\hline & 11 & Vinos (vitivinícola) & 18 & 15,1 & 15,8 & 99,1 \\
\hline & 12 & Bebidas espirituosas + Otras bebidas alcohólicas. & 1 & 0,8 & 0,9 & 100,0 \\
\hline & Total & & 114 & 95,8 & 100,0 & \\
\hline Perdidos & Sistema & & 5 & 4,2 & & \\
\hline Total & & & 119 & 100,0 & & \\
\hline
\end{tabular}

FUENTE: Elaboración propia.

Cabe recordar que para la selección de la muestra se ha seguido el muestreo probabilístico estratificado proporcionado en función de la comunidad autónoma, por ello se puede constatar que algunos de los subsectores no tienen representación. No obstante los más representativos, como son el vitivinícola, oleícola o el hortofrutícola, quedarían reflejados con un número significativo, al representar el $16 \%, 10 \%$ y $29 \%$ respectivamente, lo que supone más de la mitad de las entidades cooperativas analizadas ${ }^{15}$.

Finamente, con el objetivo de medir el grado de digitalización de las cooperativas agroalimentarias que conforman la muestra, se ha creado la variable agregada "Nivel de Digitalización", definida como el sumatorio del conjunto de variables "V" recogidas en la tabla 3. Dicha variable agregada puede tomar como valor máximo $11^{16}$ y valor mínimo 0.

15. - El estudio diferenciado por estos sectores también podrá suponer una aportación del presente trabajo a los previos analizados. 16.- También se tomará como variable de referencia la "V.O. Disponibilidad de sitio/página web de la cooperativa." 


\section{Análisis y discusión de resultados}

Los resultados de investigación que se exponen a continuación, de acuerdo con la metodología propuesta en el apartado anterior, siguen el mismo orden en el que se formularon las hipótesis de trabajo y se agrupan bajo dos grandes bloques: el primero, dedicado al análisis comparativo entre la transformación digital de las cooperativas agroalimentarias y el conjunto de empresas y, el segundo, dedicado al análisis de la influencia que ejercen las variables "tamaño" y "subsector de actividad" sobre el grado de transformación digital de las cooperativas agroalimentarias.

\subsection{Transformación digital: análisis comparativo entre cooperativas agroalimen- tarias y el conjunto de empresas}

En este apartado se recogen los resultados obtenidos del análisis comparativo desarrollado para contrastar la existencia de un mayor retraso en la transformación digital de las cooperativas agroalimentarias respecto del conjunto del sector empresarial en España, tal y como se formuló en la primera hipótesis de investigación (H1).

En la tabla 7 se presentan los resultados obtenidos tras la recopilación de datos y el análisis directo de las variables seleccionadas relativas al comercio electrónico y a los servicios web ofrecidos por las cooperativas agroalimentarias que conforman la muestra. A partir de estos datos, una vez realizada su comparación con la información suministrada por el (INE, 2017) en la "Encuesta de uso de TIC y Comercio Electrónico", se recogen en la tabla 8 los resultados del estudio comparativo realizado entre el sector cooperativo agroalimentario y el conjunto de empresas en España. 


\section{Tabla 7. Cooperativas agroalimentarias 2018: Servicios web y comercio electrónico}

\begin{tabular}{|c|c|c|}
\hline Servicios web: variables seleccionadas & Total & Total \\
\hline V.0 Disponibilidad de sitio/página web de la cooperativa (1). & 66 & $55,46 \%$ \\
\hline V.1 Presentación corporativa de la entidad cooperativa (2). & 62 & $93,94 \%$ \\
\hline V.2 Recepción de pedidos o reservas online (2) & 11 & $16,67 \%$ \\
\hline V.3. Acceso a catálogos de productos o listas de precios (2). & 50 & $75,76 \%$ \\
\hline $\begin{array}{l}\text { V.4. Posibilidad de personalización y diseño de productos } \\
\text { por parte de los clientes (2). }\end{array}$ & 3 & $4,55 \%$ \\
\hline V.5 Seguimiento online de pedidos (2). & 2 & $3,03 \%$ \\
\hline V.6 Personalización de la página Web para usuarios habituales (2). & 7 & $10,61 \%$ \\
\hline $\begin{array}{l}\text { V.7 Vínculos o referencias a los perfiles de las cooperativas en } \\
\text { medios sociales (2). }\end{array}$ & 23 & $34,85 \%$ \\
\hline $\begin{array}{l}\text { V.8 Declaración de política de intimidad o certificación relacionada } \\
\text { con la seguridad del sitio Web (2). }\end{array}$ & 41 & $62,12 \%$ \\
\hline $\begin{array}{l}\text { V.9 Anuncios de ofertas de trabajo o recepción de solicitudes } \\
\text { de trabajo online (2). }\end{array}$ & 6 & $9,09 \%$ \\
\hline V.10 Posibilidad de envío electrónico de hojas de reclamaciones (2). & 0 & $0 \%$ \\
\hline
\end{tabular}

(1) Datos sobre el total de empresas cooperativas analizadas. (2) Datos sobre el total de empresas cooperativas con página Web.

FUENTE: Elaboración propia. 


\section{Tabla 8. Transformación digitalk servicios web y comercio electrónico. Análisis comparativo entre cooperativas agroalimentarias y conjunto de empresas. España (2017-2018)}

\begin{tabular}{|c|c|c|c|c|}
\hline \multicolumn{2}{|c|}{ Variables } & \multirow{2}{*}{$\begin{array}{c}\begin{array}{c}\text { Total } \\
\text { Empresas }\end{array} \\
77,69 \%\end{array}$} & \multirow{2}{*}{$\begin{array}{c}\begin{array}{c}\text { Total } \\
\text { Cooperativas } \\
\text { agroalimentarias }\end{array} \\
55,46 \%\end{array}$} & \multirow{2}{*}{$\begin{array}{r}\text { Diferencial } \\
-22,23 \%\end{array}$} \\
\hline V.0 & $\begin{array}{l}\text { Disponibilidad de sitio/página web de la cooperativa / } \\
\text { empresa (1). }\end{array}$ & & & \\
\hline V.1 & $\begin{array}{l}\text { Presentación corporativa de la entidad cooperativa / } \\
\text { empresa (2). }\end{array}$ & $89,57 \%$ & $93,94 \%$ & $4,37 \%$ \\
\hline V.2 & Recepción de pedidos o reservas online (2). & $18 \%$ & $16,67 \%$ & $-1,33 \%$ \\
\hline V.3. & Acceso a catálogos de productos o listas de precios (2). & $49,42 \%$ & $75,76 \%$ & $26,34 \%$ \\
\hline V.4. & $\begin{array}{l}\text { Posibilidad de personalización y diseño de productos por } \\
\text { parte de los clientes (2). }\end{array}$ & $8,15 \%$ & $4,55 \%$ & $-3,60 \%$ \\
\hline V.5 & Seguimiento online de pedidos (2). & $10,9 \%$ & $3,03 \%$ & $-7,87 \%$ \\
\hline V.6 & $\begin{array}{l}\text { Personalización de la página Web para usuarios } \\
\text { habituales (2). }\end{array}$ & $7,83 \%$ & $10,61 \%$ & $2,78 \%$ \\
\hline V.7 & $\begin{array}{l}\text { Vínculos o referencias a los perfiles de las cooperativas } \\
\text { en medios sociales (2). }\end{array}$ & $46,72 \%$ & $34,85 \%$ & $-11,87 \%$ \\
\hline V.8 & $\begin{array}{l}\text { Declaración de política de intimidad o certificación } \\
\text { relacionada con la seguridad del sitio Web (2). }\end{array}$ & $71,08 \%$ & $62,12 \%$ & $-8,96 \%$ \\
\hline V.9 & $\begin{array}{l}\text { Anuncios de ofertas de trabajo o recepción de solicitudes } \\
\text { de trabajo online (2). }\end{array}$ & $21,01 \%$ & $9,09 \%$ & $-11,92 \%$ \\
\hline V.10 & $\begin{array}{l}\text { Posibilidad de envío electrónico de hojas de } \\
\text { reclamaciones (2). }\end{array}$ & $27,78 \%$ & $0 \%$ & $-27,78 \%$ \\
\hline
\end{tabular}

(1) Porcentaje sobre el total de empresas con conexión a Internet.

(2) Porcentaje sobre el total de empresas con conexión a Internet y página Web.

FUENTE: Elaboración propia a partir de INE (2017). 


\section{Figura 1. Transformación digital: servicios web y comercio electrónico. Análisis comparativo entre cooperativas agroalimentarias y conjunto de empresas. España (2017-2018)}

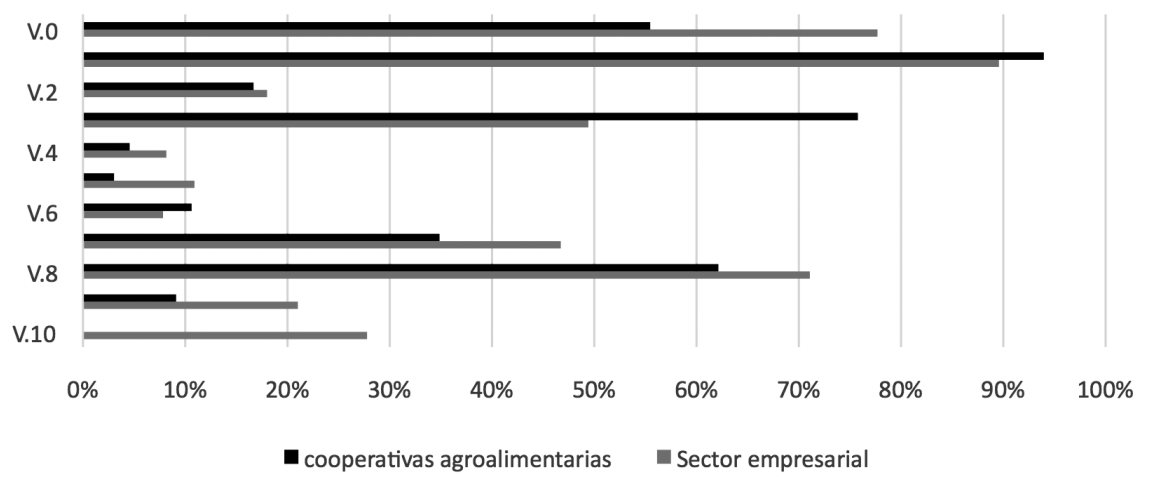

(1) Porcentaje sobre el total de empresas con conexión a Internet

(2) Porcentaje sobre el total de empresas con conexión a Internet y página Web

FUENTE: Elaboración propia a partir de INE (2017).

El análisis realizado refleja que tan sólo el $55,46 \%$ de las cooperativas agroalimentarias objeto de estudio dispone de una página web corporativa ${ }^{17}$. Dicho resultado, al compararlo con el conjunto de empresas -cuyo porcentaje se eleva al 77,69\%-, pone en evidencia la existencia de cierto retraso del sector cooperativo agroalimentario en términos de presencia en internet respecto al resultado medio alcanzado por el sector empresarial español. Obsérvese la existencia de un diferencial superior a 22 puntos porcentuales.

Del conjunto de variables analizadas, solamente tres (presentación corporativa de la empresa (V1), acceso al catálogo de productos y listas de precios (V3) y personalización de la página web para usuarios habituales (V6)) muestran un posicionamiento mejor de las cooperativas agroalimentarias, y especialmente significativo en el acceso a catálogos de productos y listas de precios. De entre ellas, cabe destacar que las empresas cooperativas definen en un 4,4\% más quienes son, lo cual podría estar en línea con su propia actividad. Sin embargo, el apartado que más diferencial presenta, con más de un $26 \%$, es el de acceso a catálogos de productos o a listas de precios, lo que contrasta con su peor posi-

17.- De este porcentaje quedan excluidas aquellas entidades cooperativas de la muestra que, a pesar de disponer de dominio propio, presentan un acceso defectuoso a sus respectivos portales web. Dichas cooperativas representan un porcentaje no superior al $1,7 \%$ de la muestra analizada. 
cionamiento en cuanto a los servicios de recepción de pedidos o reservas online. Finalmente, la personalización de la página Web, es mejor en el caso de las cooperativas agroalimentarias, aunque con un diferencial poco significativo no superior al $2,8 \%$.

En el otro extremo, y atendiendo al resto de variables, cabe destacar la inexistencia de canales electrónicos para el envío de reclamaciones dentro de los servicios web ofrecidos por las cooperativas agroalimentarias de la muestra. Por otro lado, el porcentaje de cooperativas con presencia en redes sociales y con disponibilidad de algún canal web habilitado para el reclutamiento de personal es inferior al conjunto de empresas, presentando en ambos casos un diferencial próximo a 12 puntos porcentuales. En el resto de variables analizadas las diferencias no resultan tan pronunciadas.

Los resultados obtenidos se encuentran en línea con los publicados por otros trabajos (Meroño y Arcas, 2006; Bernal y Mozas, 2008; Mozas y Bernal, 2013; Montegut, et al., 2013; Fernández et al., 2016) que han estudiado, de manera aislada y en distintos subsectores de actividad, alguno de los parámetros observados en la presente investigación sobre el sector cooperativo agroalimentario.

\subsection{La influencia de las variables tamaño y subsector de actividad sobre la trans- formación digital de las cooperativas agroalimentarias}

Con la finalidad de contrastar la segunda y tercera hipótesis y verificar si las variables tamaño y subsector de actividad, ejercen alguna influencia sobre el grado de transformación digital de las cooperativas agroalimentarias, se procede a desarrollar el correspondiente análisis de correlaciones, tal y como se recoge en las tablas siguientes.

Adicionalmente se incluye un análisis interterritorial para completar el análisis. No obstante, dado que en este aspecto la muestra para algunas comunidades no es elevada, tabla 2 , se deja para futuras investigaciones profundizar en esta línea.

En primer lugar, para determinar el "tamaño o dimensión de la empresa cooperativa" se ha optado, tal y como se apuntó con anterioridad, por emplear las siguientes variables: Ingresos explotación (A.0), socios/número de empleados (A.1) y total activos (A.2). De los cuales también se presentan los estadísticos descriptivos en la tabla 9. Se añaden el Nivel de digitalización que es la variable artificial creada como sumatorio de los ítems analizados. 


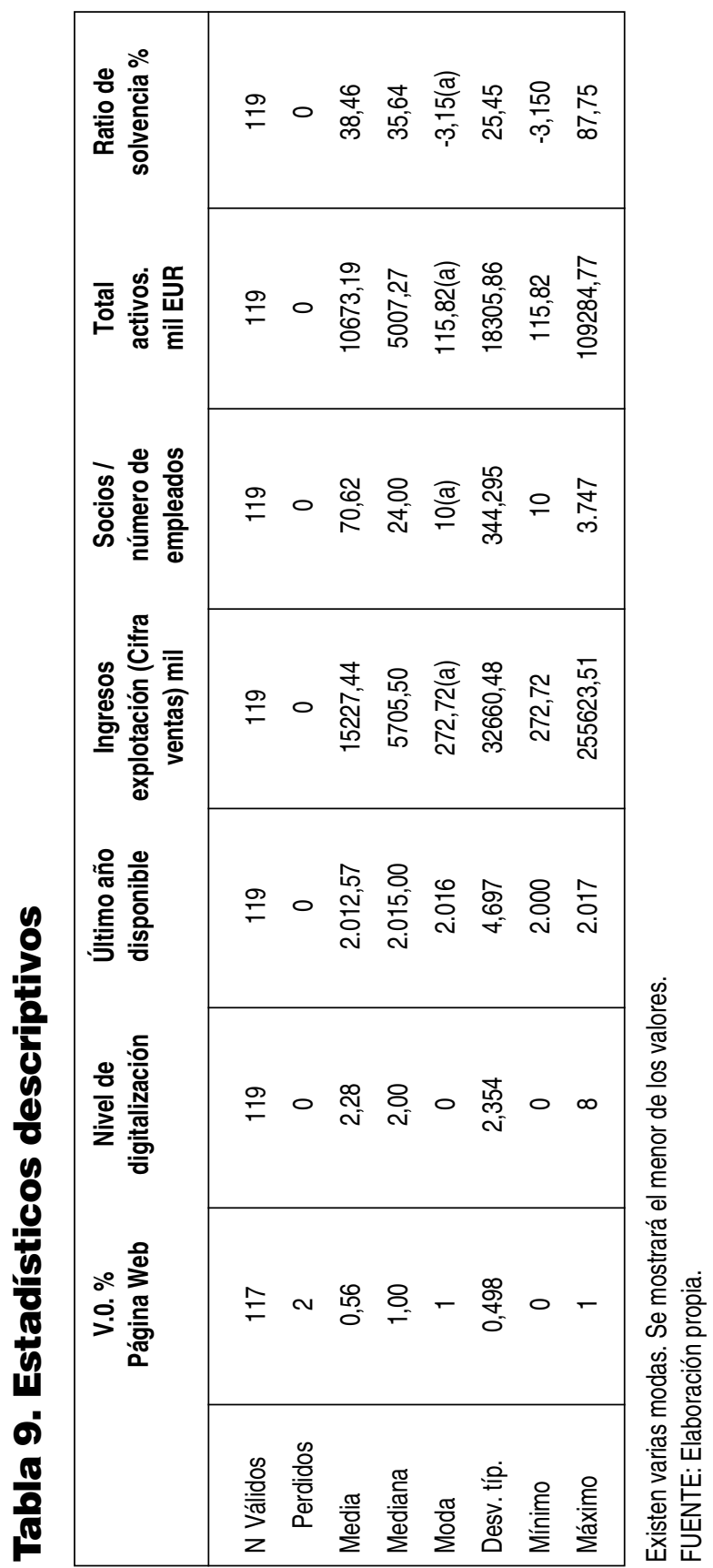


Lo más significativo es comprobar que la media en el nivel de digitalización es del 2,3 sobre 11 siendo el máximo valor que podía alcanzar. Es decir, en una escala del 0 al 10 no llegaría al aprobado. De hecho, ninguna cooperativa tiene la máxima puntuación, el mayor valor que obtienen es un 8. La media nos hace intuir que aún queda mucho por hacer respecto del nivel de transformación digital.

Tal y como se señaló, la muestra está compuesto por cooperativas con más de 10 socios/trabajadores, obteniendo una media de 70,62 socios, pero ciertamente la desviación típica también es bastante elevada con un 344. Esto viene propiciado por la existencia dentro de la muestra de sociedades cooperativas que cuentan con un importante número de socios/trabajadores ${ }^{18}$.

Adicionalmente se ha incluido la variable "Ratio de Solvencia", de manera que se comprueba que aquellas cooperativas de mayor tamaño y mayor número de socios / empleados tienen menor solvencia, es decir más endeudamiento respecto de los recursos propios. Esto podría estar propiciado por una mayor necesidad o posibilidad de endeudamiento externo al tener mayor tamaño.

Por otro lado, como se puede comprobar en la tabla 10, las variables que presentan un mayor nivel de correlación con las variables que miden el nivel de transformación digital, es decir la existencia de página web, así como la variable creada "Nivel de Digitalización", son: Ingresos explotación, socios / número de empleados y total activos. Por tanto, las tres variables establecidas para medir el tamaño de la cooperativa influyen en que estas tengan página web, y en el nivel de transformación digital, medido a través de los 10 ítems adicionales respecto a los servicios ofrecidos en internet. 


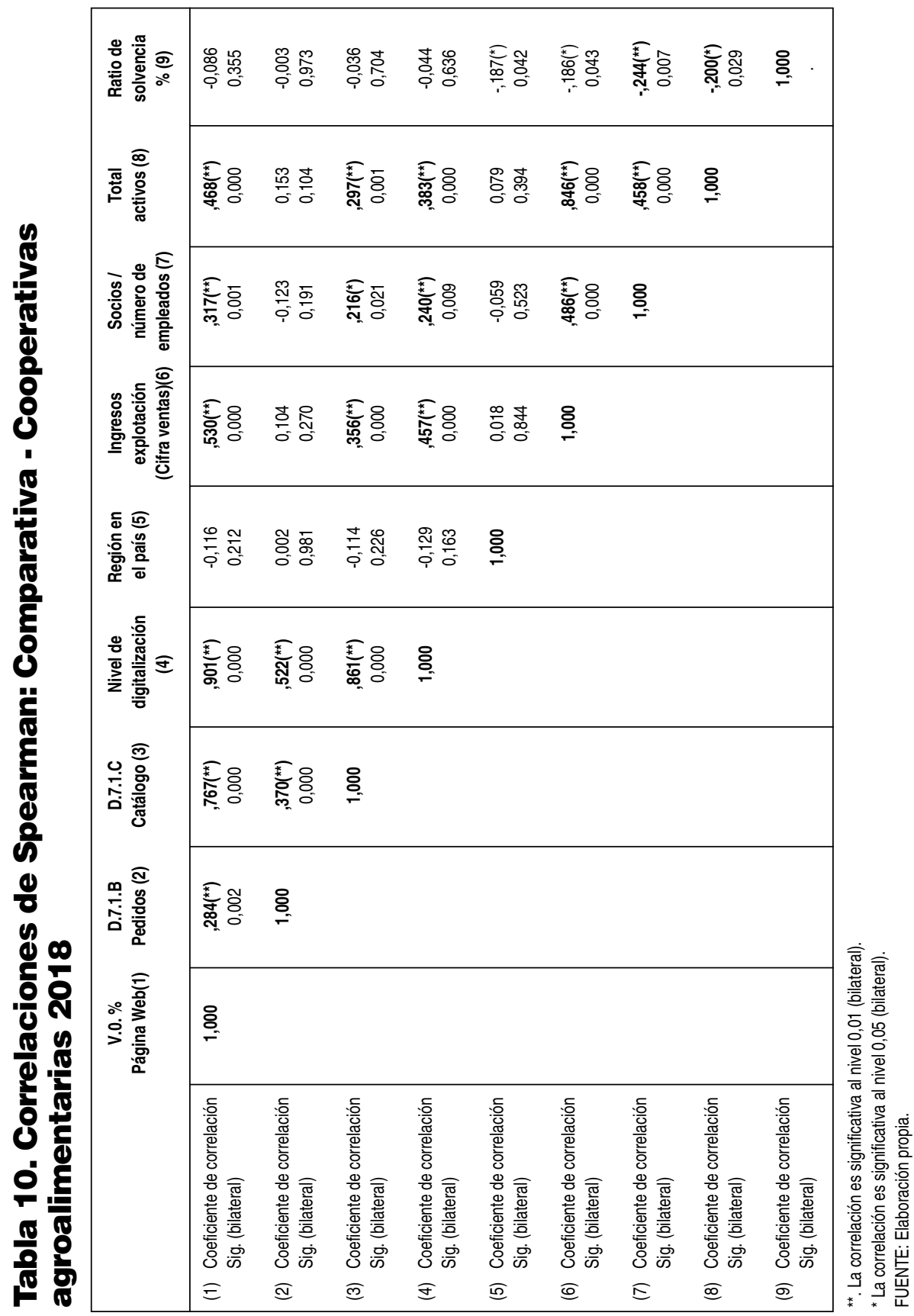


Como era de esperar también existe correlación entre las tres variables que miden el tamaño: total activos, ingresos y número de empleados.

Con la finalidad de desarrollar un análisis interterritorial y verificar si la ubicación donde desarrollan su actividad las cooperativas agroalimentarias ejerce una influencia sobre su nivel de transformación digital, se ha optado por incluir de modo adicional una variable denominada "Región del país", pero no aparecen correlaciones. Este resultado puede venir propiciado por ser una variable creada de forma artificial, dando un valor a cada región. Para evitar este problema, también se crearon variables Dummy por comunidad autónoma, para verificar si alguna presentaba una digitalización más significativa que otra, pero no se han encontrado ${ }^{19}$. Lo que si se observa es que las cooperativas agroalimentarias ubicadas en Castilla la Mancha presentan un mayor número de socios / empleados y mayores ingresos de explotación, o en el caso de Andalucía con mayores activos y mayores ingresos de explotación, lo que también parece estar en línea con el volumen y realidad de las cooperativas 20 .

Para contrastar la tercera hipótesis de trabajo, se ha dividido la muestra en los subsectores propuestos siguiendo la Clasificación Nacional de Actividades Económicas (CNAE-2009). En este caso se verifica, según se muestra en la tabla 11, el papel destacable del subsector "productos lácteos" cuyas cooperativas obtienen un valor medio del 4,67 en el nivel de digitalización. Por otro lado, el $100 \%$ de las cooperativas que operan en este subsector disponen de página web.

Otros subsectores que no pasan desapercibidos son el "oleícola" y los "productos para alimentación animal". En ambos casos las empresas cooperativas con página web que operan en este ámbito de actividad representan un porcentaje significativo del $82 \%$ y $71 \%$ respectivamente. En particular, si contrastamos estas cifras con la media del conjunto de empresas en España, podemos afirmar que tan solo los subsectores "productos lácteos" y "oleícola" presentan un porcentaje de empresas con página web superior a la media nacional.

Por otro lado, los subsectores de actividad que alcanzan una mayor puntuación en el índice de digitalización propuesto son, en orden decreciente, "productos lácteos" (4,67 puntos), "oleícola" (3,25 puntos), "industria cárnica" (2,92 puntos) y "vitivinícola (2,89 puntos). A pesar de que todos estos subsectores presentan una puntuación superior a la media del conjunto de cooperativas agroalimentarias analizadas, dicha calificación dista mucho de considerarse óptima, lo que señala la necesidad de impulsar medidas que intensifiquen el proceso de transformación digital del sector cooperativo agroalimentario. tra tomada (véase tabla 2). 


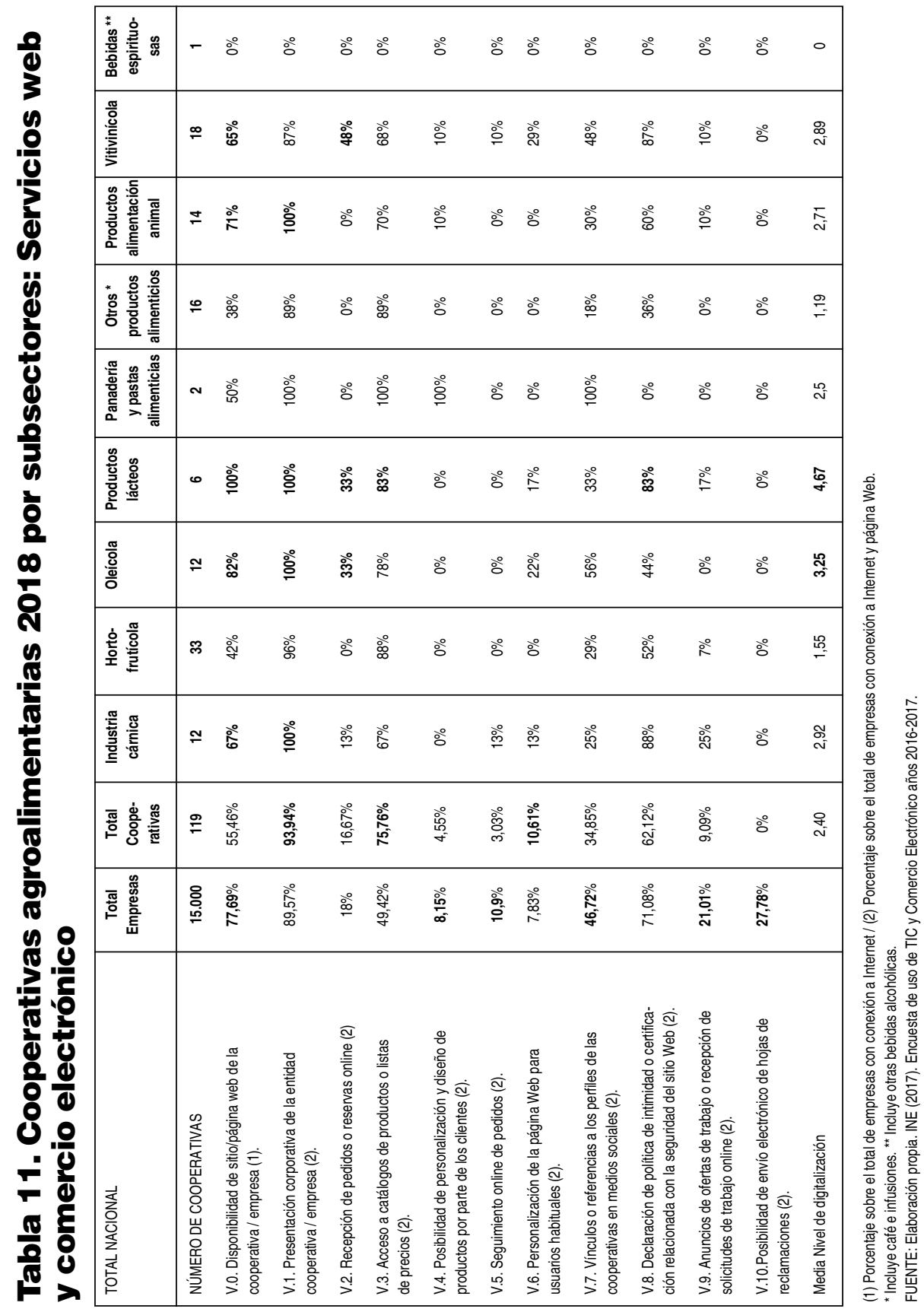


Si atendemos a las variables relacionadas con el comercio electrónico (V.2, V.3 y V.5) no se observa ningún subsector que destaque especialmente en todas ellas. Si bien el subsector "vitivinícola" despunta en la recepción de pedidos/reservas online y en el seguimiento online de pedidos, con un porcentaje muy superior a la media de las cooperativas agroalimentarias, el subsector "otros productos alimenticios" presenta un mejor acceso a catálogos de productos / listas de precios, con un valor superior a la media de cooperativas y del conjunto de empresas. A pesar de ello, dentro de los ámbitos de actividad analizados, el subsector "vitivinícola" es el que presenta un mejor grado de desarrollo en términos de comercio electrónico.

Finalmente, los subsectores con mayor presencia en redes sociales con valores por encima de la media del conjunto de cooperativas y empresas son en orden descendente "panadería y pastas alimenticias" (100\%), "oleícola" (56\%) y "vitivinícola" (48\%).

Los resultados generales obtenidos, se encuentran próximos a los alcanzados en otros estudios sobre el subsector oleícola (Mozas, et al., 2001 y 2008); Montegut et al., 2013; Uclés et al, 2016) 0 sobre el sector hortofrutícola (Meroño et al, 2006) desarrollados en determinadas regiones españolas.

\section{Conclusiones}

Con la presente investigación se ha perseguido contribuir al análisis del proceso de transformación digital del sector cooperativo agroalimentario en España, como sector estratégico del modelo productivo de nuestro país. En concreto, basándonos en el análisis de la existencia y nivel de desarrollo de los servicios web ofrecidos por dicho sector, siendo que todas las comunidades autónomas y subsectores de actividad estaban representadas en la muestra, se ha desarrollado un análisis comparativo en base a tres vertientes diferentes: interterritorial, intersectorial y interempresarial.

Los objetivos formulados han girado en torno a dos cuestiones. En primer lugar, caracterizar la situación actual sobre el grado de digitalización del sector cooperativo agroalimentario en España, así como determinar su posicionamiento relativo respecto al conjunto del sector empresarial. Y, en segundo lugar, identificar aquellos factores condicionantes que podrían ejercer una influencia relevante sobre dicho proceso de transformación digital. Asimismo, se establecían tres hipótesis de trabajo que se han ido contrastando a lo largo del estudio y de cuyos resultados se podrían extraer las siguientes conclusiones:

El nivel de digitalización de las cooperativas agroalimentarias, en términos generales, sigue siendo inferior a la media del conjunto de empresas a nivel nacional, mostrando cierto "retraso tecnológico". 


\section{LA TRANSFORMACIÓN DIGITAL EN EL SECTOR COOPERATIVO AGROALIMENTARIO ESPAÑOL: SITUACIÓN Y PERSPECTIVAS}

No obstante, este menor grado de transformación digital no es homogéneo en el conjunto de subsectores que conforman el sector agroalimentario. Así, queda constatado que el subsector de actividad ejerce una influencia significativa sobre el grado de transformación digital de la cooperativa. Así, puede observarse como el sector lácteo, oleícola, vitivinícola y la industria cárnica muestran un nivel global de digitalización superior al resto de subsectores agroalimentarios, presentando en muchas variables valores superiores a la media analizada del conjunto de empresas.

Igualmente queda demostrado que la dimensión de la cooperativa constituye un factor determinante del proceso de transformación digital de la empresa. De modo que aquellas cooperativas de mayor tamaño o con mayor volumen de recursos presentan un grado de digitalización mayor en términos de desarrollo de página web, presencia en internet, comercio electrónico y servicios web ofrecidos.

Desde el punto de vista territorial, no se ha identificado la existencia de una relación directa entre el mayor o menor grado de transformación digital de las cooperativas agroalimentarias y el territorio donde desarrollan su actividad.

Para finalizar, la disrupción digital ha supuesto un cambio significativo y acelerado en el modo de gestionar las empresas, pero a su vez, se ha convertido en una palanca que impulsa el desarrollo y la competitividad empresarial. El sector cooperativo agroalimentario, tal y como ha quedado reflejado en esta investigación, presenta, en términos globales, un nivel de digitalización subóptimo. Sin embargo, las cooperativas agroalimentarias no pueden ser ajenas a la oportunidad que brindan las nuevas tecnologías digitales y deben hacer frente a importantes desafíos empresariales y tecnológicos, como son la digitalización de la cadena de valor o los retos de la integración de las nuevas tecnologías, entre otros. De su capacidad de adaptación y aceleración del proceso de digitalización dependerá en buena parte su desarrollo futuro.

\section{Referencias bibliográficas}

ATROSTIC, B.K. \& NGUYEN, S.V. (2005): "IT and Productivity in US Manufacturing: Do Computers Networks Matter?", Economic Enquiry, 43 (3), 493-506.

BENEGAS, J. (2003): La nueva economía en España. Las TIC, la productividad y el crecimiento económico, Madrid: Alianza Editorial.

BENEGAS, J. \& MYRO, R. (2008): Impacto de las tecnologías de la información en la economía española, Madrid: Thomson-Civitas. 
BERNAL, E. \& MOZAS, A. (2008): "Evaluación del uso comercial de la World Wide Web por parte de las cooperativas de segundo grado españolas", Revista Española de Estudios Agrosociales y Pesqueros, 219, 181-200.

BERNAL, E., MOZAS, A., FERNÁNDEZ, D. \& MEDINA, M. (2017a): "Determining Factors For Economic Efficiency In The Organic Oil Sector", Sustainability, 1-9. DOI: 0.3390/su9050784.

BERNAL, E., MOZAS, A., FERNÁNDEZ, D. \& MEDINA, M. (2017b): "Explanatory factors for efficiency in the use of social networking site-The case of organic food products", Psycology And Marketing, 34, 1119-1126. DOI: 10.1002/mar.21052.

BERNAL, E., MOZAS, A., MEDINA, M. \& FERNÁNDEZ, D. (2018): "Evaluation of corporate websites and their influence on the performances of olive oil companies", Sustainability, 34, 1274-1285. DOI: 10.3390/su10041274.

BRESNAHAN, T.F., BRYNJOLFSSON, E. \& HITT, L.M. (2002): "Information Technology, Workplace Organization and the Demand for Skilled Labor: A Firm-level Evidence", Quarterly Journal of Economics, 117 (1), 339-376.

CHIVITE, M.P., ENCISO, V., GARCÍA, B. \& TÚA, J. (2014): "Determinantes de la Responsabilidad Social Corporativa: la crisis y el Efecto Contagio", CIRIEC-España, Revista de Economía Pública, Social y Cooperativa, 81, 127-161.

CONSEJO ECONÓMICO Y SOCIAL (CES) (2017): La digitalización de la economía, Madrid: Consejo Económico y Social.

ESPASANDÍN, F., CASANUEA, C. \& GANZA, J. (2004): "Las nuevas tecnologías de la información y la comunicación en la economía social andaluza", CIRIEC-España, Revista de Economía Pública, Social y Cooperativa, 49, 193-215.

EVANS, P.B. \& WURSTER, T.S. (1997): "Strategy and the new economics of information", Harvard Business Review, 75 (5), 71-82.

FERNÁNDEZ, D., BERNAL, E., MEDINA, M. \& MORAL, E. (2016): "El sector cooperativo oleícola y el uso de las TIC: un estudio comparativo respecto a otras formas jurídicas", REVESCO, Revista de Estudios Cooperativos, 120, 53-75.

GARCÍA, E., RIALP, A. \& RIALP, J. (2007): "Tecnologías de la información y comunicación (TIC) y crecimiento de la empresa", Información Comercial Española, ICE: Revista de Economía, 838, 125-145.

GHOSH, S. (1998): "Making business sense of the internet", Harvard Business Review, 126-135.

HAIR, J.F., BUSH, R.P. \& ORTINAU, D.J. (2010): Investigación de mercados en un ambiente de información digital, México: McGraw Hill. 
HERNANDO, I. \& NUÑEZ, S. (2004): "The Contribution of ICT to Economic Activity: A Growth Accounting Exercise with Spanish Firm-level Data", Investigaciones Económicas, 28 (2), 315-348.

INE (2017): Encuesta de uso de TIC y Comercio Electrónico años 2016-2017, Madrid: Instituto Nacional de Estadística.

JORGENSON, D.W. \& VU, K. (2007): "Information Technology and the world growth resurgence", German Economic Review, 8 (2), 125-145.

JULIÁ, J., GARCÍA, G. \& POLO, F. (2004): "La información divulgada a través de internet por las cooperativas", CIRIEC-España, Revista de Economía Pública, Social y Cooperativa, 49, 167-192.

LÓPEZ, E., ARCAS, N. \& ALCÓN, F. (2012): "Los sitios Web de las cooperativas agroalimentarias. Antecedentes y consecuencias de su adopción", CIRIEC-España, Revista de Economía Pública, Social y Cooperativa, 76, 261-282.

MARCUELLO, C. \& SAZ, M. (2008): "Los principios cooperativos facilitadores de la innovación: un modelo teórico, REVESCO, Revista de Estudios Cooperativos, 94, 59-79.

MAS IVARS, M. \& QUESADA IBÁÑNEZ, J. (2005): Las nuevas tecnologías y el crecimiento económico en España, Bilbao: Fundación BBVA.

MEROÑO CERDÁN, Á. \& ARCAS LARIO, N. (2006): "Equipamiento y gestión de las tecnologías de la información en las cooperatias agroalimentarias", CIRIEC-España, Revista de Economía Pública, Social y Cooperativa, 54, 5-31.

MONTEGUT SALLA, Y., CRISTÓBAL FRANSI, E. \& GÓMEZ ADILLÓN, M. (2013): "La implementación de las TIC en la gestión de las cooperativas agroalimentarias: el caso de la provincia de LLeida", REVESCO, Revista de Estudios Cooperativos, 110, 223-253.

MOZAS, A. \& BERNAL, E. (2001): "El impacto de las nuevas tecnologías en el cooperativismo agrario-agroalimentario: perspectivas de futuro", REVESCO, Revista de Estudios Cooperativos, 73, 123-147.

MOZAS, A. \& BERNAL, E. (2004): "Integración cooperativa y TIC's: presente y futuro", CIRIEC-España, Revista de Economía Pública, Social y Cooperativa, 49, 143-166.

MOZAS, A. \& BERNAL, E. (2008): "El E-Business en el cooperativismo agrario: El caso del sector oleícola", Estudios de Economía Aplicada, 1, 211-231.

MYRO, R. (2009): "Las TIC y el crecimiento de la economía española", Revista de Economía, 2, 3-13.

NORDHAUS, W. (2002): "Productivity growth and the new economy", Brooking Papers on Economic Activity, 2, 211-265.

ONTIVEROS, E. \& VIZCAÍNO, D. (2017): "La digitalización de la economía española", Información Comercial Española, ICE: Revista de economía, 898, 9-22. 
ONTSI \& FUNDETEC (2014): Análisis sectorial de implantación de las TIC en la pyme española, Madrid: Ministerio de Industria, Energía y Turismo.

PARRILLA, J.A. (2017): "La importancia de la utilización de las redes sociales en la oleicultura". En: J.A. Gómez-Limón y M. Parras Rosa, Economía y comercialización de los aceites de oliva. Factores y perspectivas para el liderazgo español del mercado global, Cajamar Caja Rural, 377395.

SEDISI (Asociación Española de Empresas de Tecnología de la Información) (2002): Las tecnologías de la sociedad de la informaicón en la empresa española 2001, SEDISI.

TORRENT, J. (2008): La empresa red. Tecnologías de la información y la comunicación, productividad y competitividad, Barcelona: Ariel.

VAN REENEN, J., DRACA, M. \& SADUN, R. (2007): "Productivity and ICTs: A Review of the Evidence. In R. Mansell", The Oxford Handbook of Information and Communication Technologies, 100-147, Oxford: Oxford University Press.

VARGAS, A. (2004): "Empresas cooperativas, ventaja competitiva y tecnologías de la información", CIRIEC-España, Revista de Economía Pública, Social y Cooperativa, 49, 13-29.

ZAMORA, A. (2016): "La economía digital, multiplicadora del crecimiento económico", Harvard Deusto business review, 262, 58-64.

ZAMORA, A. \& ARRUFÍ, J. (2017): Digital Economic Opportunity in Spain. How digitalization may boost the Spanish Economy, Accenture. 